\title{
沙冬青根瘤菌的抗逆性
}

\author{
何恒斌 1 贾昆峰 ${ }^{2}$ 贾桂霞 ${ }^{*}$ 丁 琼 ${ }^{3}$ \\ （1 北京林业大学林木花卉遗传育种重点实验室，国家花卉工程技术中心, 北京 100083）
}

（2 内蒙古乌拉特后旗林业局, 乌拉特后旗 015500）（3 中国科学院植物研究所数量植被生态学重点实验室, 北京 100093）

摘 要 沙冬青 (Ammopiptanthus mongolicus) 是西北荒漠地区珍稀常绿阔叶灌木, 综合沙冬青分布区内气候、土壤和 水分等因子, 选取宁夏中卫县沙坡头、内蒙古磴口县、内蒙古阿拉善左旗、内蒙古乌拉特后旗作为样区, 于 2004 年 4 月中下旬沙冬青的花期调查和收集根瘤, 并结合地理环境对分离得到的根瘤菌进行抗逆性初步分析。调查发现根 瘤的生成及其在宿主植物上的着生部位、大小、形状、颜色等因生境条件的不同而有所差异, 水分是其主要的影响 因子; 分离得到根瘤菌 17 株, 对其耐盐性、耐酸碱性、生长温度范围和抗生素抗性进行了研究。结果表明, 64.7\%的 菌株可以在含 $3 \% \mathrm{NaCl}$ 的 YMA 培养基上生长; $94.1 \%$ 的菌株可以在 $\mathrm{pH} 5 \sim 11$ 的范围内生长; 全部菌株在 $60{ }^{\circ} \mathrm{C}$ 处理 $10 \mathrm{~min}$ 后仍能生长; 不同菌株对不同抗生素表现出不同的抗性, $\mathrm{ZW}_{4}$ 和 $\mathrm{WH}_{4}{ }^{1}$ 对各抗生素表现出较强的抗性。分离 自磴口县的根瘤菌普遍表现出较强的抗酸碱和抗高温的能力, 这是对其环境的适应。本研究证明沙冬青根瘤菌具 有较强的抗逆性, 但菌株间存在差异, 这可能是对沙冬青分布区多种景观生态类型的适应。同时测定了几种伴生 植物中间锦鸡儿(Caragana intermedia)、猫头刺 (Oxytropis aciphylla) 和柠条 (Caragana corshinskii) 根瘤菌的抗逆性, 与沙 冬青根瘤菌表现出一定的相似性, 具较强的抗逆性, 说明生态环境对豆科植物-根瘤菌共生体有重要的影响。沙冬 青的高抗性可能与沙冬青根瘤菌的高抗性有关。沙冬青与其根瘤菌的共生关系有利于其植物群落的发育和多样 性的维系。

关键词 沙冬青 根瘤菌 抗逆性 生态环境 高抗性

\section{A PRELIMINARY STUDY ON THE STRESS RESISTANCE OF RHIZOBIA ISOLATED FROM AMMOPIPTANTHUS MONGOLICUS}

\author{
HE Heng-Bin ${ }^{1}$ JIA Kun-Feng ${ }^{2}$ JIA Gui-Xia ${ }^{1}$ and DING Qiong ${ }^{3}$ \\ (1 Laboratory of Genetics and Breeding of Trees and Flowers, National Engineering Research Center for flowers, \\ Beijing Forestry University, Beijing 100083, China) \\ ( 2 Forestry Bureau of Wulatehouqi County, Wulatehouqi, Inner Mongolia 015500, China) \\ (3 Institute of Botany, Chinese Academy of Sciences, Beijing 100093, China)
}

\begin{abstract}
Ammopiptanthus mongolicus is an endangered evergreen broad-leaf species in the northwest desert zone of China. Shapotou, Alashanzuoqi, Dengkou and Wulatehouqi were defined as research regions based on soil, water and other ecological factors. Nodules of $A$. mongolicus were collected during the last twenty-days of April, which was the flowering period of the host, and their characteristics were studied. The resistance of rhizobia isolated from A. mongolicus also was studied in relation to environmental factors of the distribution areas. Seventeen rhizobia strains were isolated from A. mongolicus. It was found that nodules had various attachment modes, sizes, shapes and colors, which were related to differences of their environment, of which water may be the most important determinant. Several biochemical characteristics were determined, including resistance to salt, acid-alkali, temperature variation and intrinsic antibiotics. The results indicated that $64.7 \%$ strains could tolerate $\mathrm{NaCl}$ stress at $3 \%$ concentration, $94.1 \%$ strains could grow at a range of $\mathrm{pH}$ from 5-11, and all strains could grow after being exposed to $60{ }^{\circ} \mathrm{C}$ for $10 \mathrm{~min}$. Differences in resistance to different intrinsic antibiotics existed among strain $\mathrm{ZW}_{4}$, and $\mathrm{Wh}_{4}{ }^{1}$ had high a resistance to different intrinsic antibiotics. Rhizobia strains from Dengkou had higher resistance to acid-alkali conditions and temperature. These results indicated that rhizobia strains isolated from A. mongolicus were highly resistant to stresses. However, there were differences among strains in their resistance to stresses, which might be related to adaptations of rhizobia to the diverse landscapes in different regions. Resistances of rhizobia strains isolated from accompanying plants, for example, Caragana corshinskii, Caragana intermedia and Oxytropis aciphylla, also
\end{abstract}


were studied, and the results indicated that they also were highly resistant to stresses similar to the rhizobia strains isolated from A. mongolicus. These results indicated that the symbiosis between rhizobia and legumes was influenced by their environment. The high resistance of $A$. mongolicus may be related to the high resistance of its rhizobia, and that the symbiotic relation was beneficial to the development and maintenance of its community in diverse landscapes.

Key words Ammopiptanthus mongolicus, Rhizobia, Resistance, Eco-environment, High resistance

根瘤菌是与豆科植物结瘤的共生固氮细菌的总 称。目前已知能够与豆科植物结瘤的细菌约有 40 个种, 均为革兰氏阴性细菌, 属于细菌域 (Bacteria)、 变形杆菌门(Proteobacteria)。根瘤菌与豆科植物形 成的根瘤或茎瘤固氮共生体系在农、林、牧业的可持 续发展中具有重要作用。在全世界已知的约 650 属 19000 多种 (Allen \& Allen, 1981) 豆科植物中, 只有约 $15 \%$ 的种做过结瘤的调查研究, 其中 $0.3 \% \sim 0.5 \%$ 研究过共生关系 (Rao Subba, 1999)。对豆科植物与 根瘤菌共生关系的认识还不完善。100 多年前, 在 根瘤菌研究的起始阶段, 人们提出的“宿主专一性” 及“互接种族”两个概念一直流传至今。 20 世纪 40 年代, 有学者根据实验室内豆科植物的交叉结瘤结 果否定过“互接种族”的观念, 70 80 年代又发现某 些根瘤菌在实验室条件下有广谱共生现象。但根瘤 菌与豆科植物共生体与地理环境的关系却很少被涉 及过。

沙冬青 (Ammopiptanthus mongolicus) 是生长在西 北荒漠地区的常绿阔叶灌木, 为第三纪子遗种, 被国 家列为首批重点保护的珍稀濒危植物 (刘果厚, 1998)。沙冬青是优良的固沙植物, 具有抗热、抗旱、 抗寒、耐盐碱、耐贫瘦、耐沙埋、抗风蚀等特性(中国 植物志编辑委员会, 1998), 且地上部分分枝多, 冠幅 较大, 阻沙能力强。正因为沙冬青具有这些优良特 性, 不少学者对沙冬青的植物群落、濒危原因、抗逆 生理、生理生态、超微结构等方面做了大量的研究 刘家琼等, 1995; 王庆锁等, 1995; 周宜君等, 2001; 陈 䂀等, 2002)。沙冬青作为一种豆科植物与根瘤菌建 立共生关系, 在西北荒漠地区缺水少氮的环境条件 下, 这种共生关系对沙冬青的生长发育、优良性状的 形成及其生物群落的发育和多样性的维系具有重要 作用。我们在沙冬青的分布区内综合各种生态因 子,选取 4 个样区对沙冬青根瘤菌进行了资源调查 和收集, 并结合地理环境对其抗逆性初步分析。

\section{1 材料和方法}

\section{1 根瘤菌的资源调查和收集}

综合分布区内气候、土壤和水分等条件,我们选
定宁夏中卫县沙坡头 $\left(37^{\circ} 32^{\prime} \mathrm{N}, 105^{\circ} 02^{\prime} \mathrm{E}\right)$ 、内蒙古 阿拉善左旗 $\left(37^{\circ} 24^{\prime} \sim 41^{\circ} 52^{\prime} \mathrm{N}\right.$; $103^{\circ} 21^{\prime} \sim 106^{\circ} 51^{\prime}$ $\mathrm{E}) 、$ 蒙古磴口县 $\left(40^{\circ} 13^{\prime} \mathrm{N}, 107^{\circ} 05^{\prime} \mathrm{E}\right)$ 、内蒙古乌拉 特后旗 $\left(41^{\circ} 43^{\prime} \mathrm{N}, 106^{\circ} 22^{\prime} \mathrm{E}\right.$ ) 4 个地区进行固定采 样, 并根据样区内的地形和土壤等条件选取采样点。 将沙冬青和其豆科伴生植物的根系挖出, 收集根瘤, 编号; 并记录采样时间、地点、生态植被、土壤类型、 水分条件等。

\section{2 根瘤菌的分离}

根瘤菌的分离和纯化采用的培养基为 YMA 培 养基, 参照陈文新等(2004) 的方法加以配制。分离 纯化参照 Chen 等(1991)的方法。分离得到的菌株 保存于超低温冰箱中备用。

\section{3 根瘤菌的回接}

将经过活化的根瘤菌菌株制成菌悬液倒入培养 皿中, 将沙冬青发芽的种子在其中浸泡 30 45 min, 后植入装有沙冬青培养基质的容器中, 并定时施加 菌悬液。

\section{4 抗逆性测定}

\subsection{1 耐盐性测定}

将试验菌株进行活化培养, 将培养的菌株在含 有 $\mathrm{NaCl}$ 的平板上划板, $\mathrm{NaCl}$ 的最终浓度依次为 $1 \%, 2 \%, 3 \%, 4 \%, 5 \%, 6 \%, 7 \%$, 以含 $0.01 \% \mathrm{NaCl}$ 的 YMA 划线平板作为阳性对照, $28{ }^{\circ} \mathrm{C}$ 培养观察 $3 \sim$ $5 \mathrm{~d}$, 生长记为“+”, 不生长记为“ - ”。

\subsection{2 耐酸碱性测定}

高压灭菌的 YMA 培养基用 $\mathrm{HCl}$ 和 $\mathrm{NaOH}$ 调节 $\mathrm{pH}$ 值, $\mathrm{pH}$ 值依次为 3.0、4.0、5.0、6.0、8.0、9.0、 $10.0 、 11.0 、 12.0$, 以 $\mathrm{pH} 7.0$ 的划线平板作为阳性对 照, 培养, 观察记录同上。

\section{4 .3 生长温度范围测定}

将供试菌株接种于 YMA 培养基上, 分别在 4、 12、20、37、45 ${ }^{\circ} \mathrm{C}$ 下进行培养; 分别将菌株在 50 和 60 ${ }^{\circ} \mathrm{C}$ 下处理 $10 \mathrm{~min}$ 后, 在 $28{ }^{\circ} \mathrm{C}$ 下培养。以 $28{ }^{\circ} \mathrm{C}$ 的 YMA 划线平板为阳性对照, 观察 $7 \sim 10 \mathrm{~d}$, 记录同 上。

1.4.4 抗生素抗性测定

选取卡那霉素 $(K m)$ 、氨苄青霉素 $(A m p)$ 、氯霉 
素 $(\mathrm{Cm})$ 和四环素 $(\mathrm{Tc}) 4$ 种抗生素, 每种抗生素 4 个 浓度: 5、50、100 和 $300 \mu \mathrm{g} \cdot \mathrm{ml}^{-1}$ 。将各种浓度的抗生 素溶液过滤灭菌后, 分别加入到高温灭菌后的 YMA 培养基中, 制成平板, 然后进行供试菌株的划线接 种。以不含抗生素 YMA 培养基作为阳性对照, 28 ${ }^{\circ} \mathrm{C}$ 培养 $7 \mathrm{~d}$, 观察记录同上。

以上每个处理均 3 次重复, 参比菌株 (CAN) 由 中国农业大学提供。

\section{2 结果与分析}

2.1 结瘤调查和根瘤特性

调查发现, 根瘤在降雨量相对较多的沙坡头和
地下水位较高的磴口比较容易采到; 大部分根瘤采 于沙冬青的开花期 4 月下旬, 7 月份根瘤已全部消 解。

根瘤的形状多种多样, 以杆状最多, 还有圆形、 椭圆形、珊瑚状和姜状; 大小 $1 \sim 15 \mathrm{~mm}$ 不等; 颜色一 般为淡黄色或褐色。根瘤多见于侧根和须根, 少见 于主根; 主要集中于 $10 \sim 50 \mathrm{~cm}$ 的土层。

\section{2 根瘤菌回接结瘤情况}

对采集的根瘤进行分离纯化, 得到沙冬青根瘤 菌菌株 17 个, 沙冬青豆科伴生植物柠条 (Caragana korshinskii) 菌株 2 个, 锦鸡儿 ( C. intermedia) 菌株 1 个, 猫头刺 (Oxytropis aciphylla)菌株 1 个(见表 1)。

表 1 试验菌株采集地点及参比菌株来源

Table 1 Sampling places of the experimental strains and the source of the reference strain

\begin{tabular}{|c|c|c|c|}
\hline 菌株号 Strains number & 宿主名 Host & 采集地点 Sampling places & 地形及土壤 Terrain and soil \\
\hline $\mathrm{DK}_{1}{ }^{1}$ & $\begin{array}{l}\text { 沙冬青 } \\
\text { Ammopiptanthus mongolicus }\end{array}$ & 磴口 Dengkou & $\begin{array}{l}\text { 平坦风蚀沙地, 风沙土加粘土 Wind erosion moving sand, } \\
\text { windy sand soil and clay }\end{array}$ \\
\hline $\mathrm{DK}_{1}^{2}$ & 沙冬青 A. mongolicus & 磴口 Dengkou & $\begin{array}{l}\text { 平坦风蚀沙地, 风沙土加粘土 Wind erosion moving sand, } \\
\text { windy sand soil and clay }\end{array}$ \\
\hline $\mathrm{DK}_{4}$ & 沙冬青 A. mongolicus & 磴口 Dengkou & $\begin{array}{l}\text { 盐渍化滩地, 粗砾质风沙土 Saline - alkaline land, windy } \\
\text { stone - sand soil }\end{array}$ \\
\hline $\mathrm{DK}_{5}$ & 沙冬青 A. mongolicus & 磴口 Dengkou & $\begin{array}{l}\text { 洪水冲积沟滩地, 粉尘风沙土 Land caused by seasonal } \\
\text { floods, windy sand soil }\end{array}$ \\
\hline $\mathrm{DK}_{6}$ & 沙冬青 A. mongolicus & 磴口 Dengkou & $\begin{array}{l}\text { 洪水冲积沟滩地, 粉尘风沙土 Land caused by seasonal } \\
\text { floods, windy sand soil }\end{array}$ \\
\hline $\mathrm{DK}_{8}$ & 沙冬青 A. mongolicus & 磴口 Dengkou & $\begin{array}{l}\text { 盐渍化滩地, 粗砾质风沙土 Saline - alkaline land, windy } \\
\text { stone - sand soil }\end{array}$ \\
\hline $\mathrm{ZW}_{4}$ & 沙冬青 A. mongolicus & 中卫沙坡头 Shapotou & 低山坡地, 红土质灰棕漠土 Hillside, grey brown desert soil \\
\hline $\mathrm{ZW}_{7}$ & 沙冬青 A. mongolicus & 中卫沙坡头 Shapotou & 低山坡地,红土质灰棕漠土 Hillside, grey brown desert soil \\
\hline $\mathrm{ZW}_{8}$ & 沙冬青 A. mongolicus & 中卫沙坡头 Shapotou & 低山坡地,红土质灰棕漠土 Hillside, grey brown desert soil \\
\hline $\mathrm{WH}_{1}{ }^{1}$ & 沙冬青 A. mongolicus & 乌拉特后旗 Wulatehouqi & $\begin{array}{l}\text { 平坦石质滩地及沙丘, 风沙土和红土质灰棕漠土 Flood } \\
\text { land and dune, windy sand soil and grey brown desert soil }\end{array}$ \\
\hline $\mathrm{WH}_{1}^{2}$ & 沙冬青 A. mongolicus & 乌拉特后旗 Wulatehouqi & $\begin{array}{l}\text { 平坦石质滩地及沙丘, 风沙土和红土质灰棕漠土 Flood } \\
\text { land and dune, windy sand soil and grey brown desert soil }\end{array}$ \\
\hline $\mathrm{WH}_{1}^{3}$ & 沙冬青 A. mongolicus & 乌拉特后旗 Wulatehouqi & $\begin{array}{l}\text { 平坦石质滩地及沙丘, 风沙土和红土质灰棕漠土 Flood } \\
\text { land and dune, windy sand soil and grey brown desert soil }\end{array}$ \\
\hline $\mathrm{WH}_{4}{ }^{1}$ & 沙冬青 A. mongolicus & 乌拉特后旗 Wulatehouqi & $\begin{array}{l}\text { 平坦石质滩地及沙丘, 风沙土和红土质灰棕漠土 Flood } \\
\text { land and dune, windy sand soil and grey brown desert soil }\end{array}$ \\
\hline $\mathrm{WH}_{4}^{2}$ & 沙冬青 A. mongolicus & 乌拉特后旗 Wulatehouqi & $\begin{array}{l}\text { 平坦石质滩地及沙丘, 风沙土和红土质灰棕漠土 Flood } \\
\text { land and dune, windy sand soil and grey brown desert soil }\end{array}$ \\
\hline $\mathrm{WH}_{6}$ & 沙冬青 A. mongolicus & 乌拉特后旗 Wulatehouqi & $\begin{array}{l}\text { 平坦石质滩地及沙丘, 凤沙土和红土质灰棕漠土 Flood } \\
\text { land and dune, windy sand soil and grey brown desert soil }\end{array}$ \\
\hline $\mathrm{AZ}_{1}$ & 沙冬青 A. mongolicus & 阿拉善左旗 Alashanzuoqi & 低山残塬, 红土质灰棕漠土 Loess hill, grey brown desert soil \\
\hline $\mathrm{AZ}_{2}$ & 沙冬青 A. mongolicus & 阿拉善左旗 Alashanzuoqi & 残存河床, 风沙土 Riverbed， windy sand soil \\
\hline $\begin{array}{l}\text { CAN (参 比菌株 } \\
\text { Reference strain) }\end{array}$ & 沙冬青 A. mongolicus & 磴口 Dengkou & 购于中国农业大学 Bought from China Agriculture Universituy \\
\hline WHn & 柠条 Caragana korshinskii & 乌拉特后旗 Wulatehouqi & $\begin{array}{l}\text { 平坦石质滩地及沙丘, 风沙土和红土质灰棕漠土 Flood } \\
\text { land and dune, windy sand soil and grey brown desert soil }\end{array}$ \\
\hline $\mathrm{WHj}$ & 锦鸡儿 $C$. intermedia & 乌拉特后旗 Wulatehouqi & $\begin{array}{l}\text { 平坦石质滩地及沙丘, 风沙土和红土质灰棕漠土 Flood } \\
\text { land and dune, windy sand soil and grey brown desert soil }\end{array}$ \\
\hline $\mathrm{ZWn}$ & 柠条 C. korshinskii & 中卫沙坡头 Shapotou & 低山坡地, 红土质灰棕漠土 Hillside, grey brown desert soil \\
\hline WHm & 猫头刺 Oxytropis aciphylla & 乌拉特后旗 Wulatehouqi & $\begin{array}{l}\text { 平坦石质滩地及沙丘, 风沙土和红土质灰棕漠土 Flood } \\
\text { land and dune, windy sand soil and grey brown desert soil }\end{array}$ \\
\hline
\end{tabular}


用分离到的根瘤菌菌株与沙冬青进行了回接试 验, 结瘤时间在 $50 \sim 70 \mathrm{~d}$, 沙冬青根瘤菌回接结瘤率 为 $87.3 \%$, 其中 $\mathrm{DK}_{1}{ }^{1}$ 和 $\mathrm{AZ}_{1}$ 回接结瘤率较高 (分别 为 $93.3 \%$ 和 $92.7 \%), \mathrm{WH}_{6}$ 较低 $(72.8 \%)$ 。其它豆 科伴生植物的根瘤菌对沙冬青的回接结瘤率较低, 但每个菌株均有结瘤, 平均为 $21.5 \%$ 。回接结瘤的 菌株, 进一步被证明是有效的根瘤菌, 将被用作根瘤 菌分类研究的原始菌株 (结果待发表)。

\section{3 抗逆性分析}

\subsection{1 耐盐性和耐酸碱性分析}

如表 2 所示, 沙冬青根瘤菌具有非常高的耐盐 性, 菌株 $\mathrm{DK}_{8} 、 Z \mathrm{ZW}_{8}$ 和 $\mathrm{AZ}_{2}$ 甚至可以在含有 $7 \%$ 的 $\mathrm{NaCl}$ 的培养基上生长, $35.3 \%$ 的沙冬青菌株可以忍 耐 $6 \%$ 的 $\mathrm{NaCl}, 64.7 \%$ 的菌株可以在含有 $3 \%$ 的 $\mathrm{NaCl}$ 的培养基上生长; 沙冬青豆科伴生植物根瘤菌的耐 盐性也较高。随着盐离子浓度的升高, 根瘤菌生活 力逐渐下降, 生长缓慢。

据报道(关桂兰等, 1991), 根瘤菌生长的最适值 为 $\mathrm{pH} 6 \sim 7$, 有些根瘤菌在 $\mathrm{pH} 4.5 \sim 9.5$ 范围内能生 长。沙冬青根瘤菌生长的 $\mathrm{pH}$ 范围非常宽。94.1\% 的沙冬青菌株可以在 $\mathrm{pH} 5 \sim 11$ 的范围内生长, 菌株 $\mathrm{DK}_{6} 、 \mathrm{DK}_{8}$ 和 $\mathrm{AZ}_{2}$ 在 $\mathrm{pH}$ 为 $4 \sim 12$ 的环境中仍有活力,

表 2 不同浓度的 $\mathbf{N a C l}$ 对根瘤菌生长的影响

Table 2 Effects of $\mathrm{NaCl}$ on growth of rhizobium

\begin{tabular}{|c|c|c|c|c|c|c|c|}
\hline \multirow{2}{*}{$\begin{array}{c}\text { 菌株 } \\
\text { Strains }\end{array}$} & \multicolumn{7}{|c|}{$\mathrm{NaCl}(\%)$} \\
\hline & 1 & 2 & 3 & 4 & 5 & 6 & 7 \\
\hline $\mathrm{DK}_{1}{ }^{1}$ & + & + & + & - & - & - & - \\
\hline $\mathrm{DK}_{1}^{2}$ & + & + & - & - & - & - & - \\
\hline $\mathrm{DK}_{4}$ & + & + & + & + & + & + & - \\
\hline $\mathrm{DK}_{5}$ & + & - & - & - & - & - & - \\
\hline $\mathrm{DK}_{6}$ & + & - & - & - & - & - & - \\
\hline $\mathrm{DK}_{8}$ & + & + & + & + & + & + & + \\
\hline $\mathrm{ZW}_{4}$ & + & + & - & - & - & - & - \\
\hline $\mathrm{ZW}_{7}$ & + & + & + & + & + & - & - \\
\hline $\mathrm{ZW}_{8}$ & + & + & + & + & + & + & + \\
\hline $\mathrm{WH}_{1}{ }^{1}$ & + & - & - & - & - & - & - \\
\hline $\mathrm{WH}_{1}{ }^{2}$ & + & - & - & - & - & - & - \\
\hline $\mathrm{WH}_{1}{ }^{3}$ & - & - & - & - & - & - & - \\
\hline $\mathrm{WH}_{4}{ }^{1}$ & + & - & - & - & - & - & - \\
\hline $\mathrm{WH}_{4}{ }^{2}$ & + & + & + & + & - & - & - \\
\hline $\mathrm{WH}_{6}$ & + & + & + & - & - & - & - \\
\hline $\mathrm{AZ}_{1}$ & - & - & - & - & - & - & - \\
\hline $\mathrm{AZ}_{2}$ & + & + & + & + & + & + & + \\
\hline CAN & + & + & + & - & - & - & - \\
\hline WHn & + & + & + & + & + & + & - \\
\hline $\mathrm{WHj}$ & + & + & + & + & + & + & - \\
\hline$Z W n$ & - & - & - & - & - & - & - \\
\hline WHm & + & + & + & + & + & + & + \\
\hline
\end{tabular}

其它菌株生长受到抑制; 沙冬青豆科伴生植物根瘤 菌的耐酸碱范围也很广泛 ( 见表 3 )。

2.3.2 温度变化对沙冬青根瘤菌生长的影响

一般认为, 根瘤菌的最适生长温度为 $25 \sim 30$ ${ }^{\circ} \mathrm{C}$, 只有少数苜宿根瘤菌菌株可以在 $42.5{ }^{\circ} \mathrm{C}$ 下生 长, 极少数根瘤菌可以在 $4{ }^{\circ} \mathrm{C}$ 条件下生长 (关桂兰 等, 1990)。根瘤菌在 $60{ }^{\circ} \mathrm{C}$ 条件下处理 5 min 即全部 被杀死(关桂兰等, 1991)。沙冬青根瘤菌对温度的 适应范围较宽, $94.1 \%$ 的菌株可以在 $12{ }^{\circ} \mathrm{C}$ 和 $37{ }^{\circ} \mathrm{C}$ 下生长, $41.2 \%$ 的菌株在 $4{ }^{\circ} \mathrm{C}$ 下仍有活力, $23.5 \%$ 的 菌株在 $45{ }^{\circ} \mathrm{C}$ 下仍能生长; $60{ }^{\circ} \mathrm{C}$ 处理 $10 \mathrm{~min}$ 后, 在 $28{ }^{\circ} \mathrm{C}$ 下培养, 全部菌株均有出活力, 表现出极强的 抗热性; 沙冬青豆科伴生植物的根瘤菌同样具有很 宽的生长温度适应范围( 见表 4)。

\subsection{3 抗生素抗性}

根瘤菌对天然抗菌素敏感性是一项重要的生理 生化特性, 而且可作为分群的一项指标。根瘤菌对 抗菌素较敏感,被抗菌素抑制(王静等, 1999)。对所 获得的 17 株沙冬青根瘤菌菌株固有抗性的测定结 果表明(见表 5), $40.9 \%$ 的菌株对 $300 \mu \mathrm{g} \cdot \mathrm{ml}^{-1}$ 的 Amp 仍有抗性, $45.5 \%$ 的菌株对 $300 \mu \mathrm{g}^{\bullet} \mathrm{ml}^{-1}$ 的 $\mathrm{Cm}$ 有抗性, $47.1 \%$ 的菌株对 $50 \mu \mathrm{g} \cdot \mathrm{ml}^{-1}$ 的 $\mathrm{Km}$ 有抗性,

表 3 不同的 $\mathbf{p H}$ 值对根瘤菌生长的影响

Table 3 Effects of $\mathrm{pH}$ on growth of rhizobium

\begin{tabular}{|c|c|c|c|c|c|c|c|c|c|c|}
\hline \multirow{2}{*}{$\begin{array}{c}\text { 菌株 } \\
\text { Strains }\end{array}$} & \multicolumn{10}{|c|}{$\mathrm{pH}$} \\
\hline & 3 & 4 & 5 & 6 & 8 & 9 & 10 & 11 & 12 & 13 \\
\hline $\mathrm{DK}_{1}{ }^{1}$ & - & + & + & + & + & + & + & + & - & - \\
\hline $\mathrm{DK}_{1}^{2}$ & - & + & + & + & + & + & + & + & - & - \\
\hline $\mathrm{DK}_{4}$ & - & - & - & + & + & + & + & + & + & - \\
\hline $\mathrm{DK}_{5}$ & - & - & + & + & + & + & + & + & - & - \\
\hline $\mathrm{DK}_{6}$ & - & + & + & + & + & + & + & + & + & - \\
\hline $\mathrm{DK}_{8}$ & - & + & + & + & + & + & + & + & + & - \\
\hline $\mathrm{ZW}_{4}$ & - & - & + & + & + & + & + & + & + & - \\
\hline $\mathrm{ZW}_{7}$ & - & - & + & + & + & + & + & + & - & - \\
\hline $\mathrm{ZW}_{8}$ & - & - & + & + & + & + & + & + & + & - \\
\hline $\mathrm{WH}_{1}{ }^{1}$ & - & - & + & + & + & + & + & + & - & - \\
\hline $\mathrm{WH}_{1}{ }^{2}$ & - & + & + & + & + & + & + & + & - & - \\
\hline $\mathrm{WH}_{1}{ }^{3}$ & - & + & + & + & + & + & + & + & - & - \\
\hline $\mathrm{WH}_{4}{ }^{1}$ & - & + & + & + & + & + & + & + & - & - \\
\hline $\mathrm{WH}_{4}{ }^{2}$ & - & - & + & + & + & + & + & + & + & - \\
\hline $\mathrm{WH}_{6}$ & - & - & + & + & + & + & + & + & - & - \\
\hline $\mathrm{AZ}_{1}$ & - & - & + & + & + & + & + & + & - & - \\
\hline $\mathrm{AZ}_{2}$ & - & + & + & + & + & + & + & + & + & - \\
\hline CAN & - & - & - & - & + & + & + & + & - & - \\
\hline WHn & - & - & - & - & + & + & + & + & + & - \\
\hline WHj & - & + & + & + & + & + & + & + & + & - \\
\hline $\mathrm{ZWn}$ & - & - & + & + & + & + & + & + & - & - \\
\hline WHm & - & + & + & + & + & + & + & + & + & - \\
\hline
\end{tabular}


表 4 温度对根瘤菌生长的影响

Table 4 Effects of temperature on growth of rhizobium

\begin{tabular}{|c|c|c|c|c|c|c|c|}
\hline \multirow{2}{*}{$\begin{array}{c}\text { 菌株 } \\
\text { Strains }\end{array}$} & \multicolumn{7}{|c|}{ 温度 Temperature $\left({ }^{\circ} \mathrm{C}\right)$} \\
\hline & 4 & 12 & 20 & 37 & 45 & $50(10 \mathrm{~min})$ & $60(10 \mathrm{~min})$ \\
\hline $\mathrm{DK}_{1}{ }^{1}$ & - & + & + & + & - & + & + \\
\hline $\mathrm{DK}_{1}^{2}$ & - & + & + & + & - & + & + \\
\hline $\mathrm{DK}_{4}$ & + & + & + & + & + & + & + \\
\hline $\mathrm{DK}_{5}$ & + & + & + & - & - & + & + \\
\hline $\mathrm{DK}_{6}$ & - & + & + & + & + & + & + \\
\hline $\mathrm{DK}_{8}$ & - & + & + & + & + & + & + \\
\hline $\mathrm{ZW}_{4}$ & + & + & + & + & - & + & + \\
\hline $\mathrm{ZW}_{7}$ & - & + & + & + & - & + & + \\
\hline $\mathrm{ZW}_{8}$ & + & + & + & + & + & + & + \\
\hline $\mathrm{WH}_{1}{ }^{1}$ & - & + & + & + & - & + & + \\
\hline $\mathrm{WH}_{1}{ }^{2}$ & - & + & + & + & - & + & + \\
\hline $\mathrm{WH}_{1}{ }^{3}$ & - & - & + & + & - & + & + \\
\hline $\mathrm{WH}_{4}{ }^{1}$ & - & + & + & + & - & + & + \\
\hline $\mathrm{WH}_{4}{ }^{2}$ & - & + & + & + & - & + & + \\
\hline $\mathrm{WH}_{6}$ & - & + & + & + & - & + & + \\
\hline $\mathrm{AZ}_{1}$ & + & + & + & + & - & + & + \\
\hline $\mathrm{AZ}_{2}$ & + & + & + & + & - & + & + \\
\hline CAN & + & + & + & + & - & + & + \\
\hline WHn & - & + & + & + & - & + & + \\
\hline WHj & - & + & + & + & - & + & + \\
\hline $\mathrm{ZWn}$ & - & - & + & - & - & + & + \\
\hline WHm & - & - & + & + & + & + & + \\
\hline
\end{tabular}

但随着抗生素浓度的增加, 培养基上正常生长的菌 株逐渐减少; 只有很少菌株对 Rif 有抗性; 不同菌株 具有不同的抗药性特征, 对抗生素的敏感性差异很 大, $\mathrm{DK}_{6} 、 \mathrm{DK}_{8} 、 \mathrm{ZW}_{7}$ 和 $\mathrm{ZW}_{8}$ 几乎对所用各浓度的抗生 素均无抗性, 而 $\mathrm{ZW}_{4}$ 和 $\mathrm{WH}_{4}{ }^{1}$ 对各种抗生素表现出 较强的抗性。

\section{3 讨 论}

许多研究结果表明, 影响根瘤菌与其宿主共生 固氮的因素较为复杂,除遗传因素起主导作用外,生 态环境也有可能成为生物生存的重要调节因素 (Allen \& Allen, 1981; Vincent, 1970; 王卫卫等, 1995; 韩素芬等, 1996; 陈文新等, 2004)。据我们的 调查和采样结果, 沙冬青群落因水分、土壤类型及地 形差异,在不同样区内成分和结构有一定变化。水 分是沙冬青植物群落外貌的决定性生态因子, 群落 丰度、盖度及高度均与降水量成正相关。随着降水 的减少和地下水位的降低, 群落逐渐变得单调, 盖度 变小, 而群落也更趋于低矮。而沙冬青及其豆科伴 生植物的根瘤量随降水的减少和地下水位的降低而 减少, 结瘤量表现为沙坡头 > 磴口 > 阿拉善左旗 >

表 5 沙冬青根瘤菌对抗生素的抗性

Table 5 The resistance of Ammopiptanthus mongolicus thtzobia to antibioties

\begin{tabular}{|c|c|c|c|c|c|c|c|c|c|c|c|c|c|c|c|c|}
\hline \multirow{2}{*}{$\begin{array}{c}\text { 菌株 } \\
\text { Strains }\end{array}$} & \multicolumn{4}{|c|}{$\mathrm{Km}\left(\mu \mathrm{g} \cdot \mathrm{ml}^{-1}\right)$} & \multicolumn{4}{|c|}{$\operatorname{Amp}\left(\mu \mathrm{g} \cdot \mathrm{ml}^{-1}\right)$} & \multicolumn{4}{|c|}{$\operatorname{Rif}\left(\mu \mathrm{g} \cdot \mathrm{ml}^{-1}\right)$} & \multicolumn{4}{|c|}{$\mathrm{Cm}\left(\mu \mathrm{g} \cdot \mathrm{ml}^{-1}\right)$} \\
\hline & 5 & 50 & 100 & 300 & 5 & 50 & 100 & 300 & 5 & 50 & 100 & 300 & 5 & 50 & 100 & 300 \\
\hline $\mathrm{DK}_{1}{ }^{1}$ & + & + & - & - & + & + & + & + & + & - & - & - & + & + & + & + \\
\hline $\mathrm{DK}_{1}^{2}$ & + & + & + & - & + & + & + & + & + & - & - & - & + & + & + & + \\
\hline $\mathrm{DK}_{4}$ & - & - & - & - & + & - & - & - & - & - & - & - & + & - & - & - \\
\hline $\mathrm{DK}_{5}$ & + & + & - & - & + & + & + & - & - & - & - & - & + & + & - & \\
\hline $\mathrm{DK}_{6}$ & - & - & - & - & - & - & - & - & - & - & - & - & + & - & - & - \\
\hline $\mathrm{DK}_{8}$ & - & - & - & - & + & - & - & - & - & - & - & - & - & - & - & - \\
\hline $\mathrm{ZW}_{4}$ & + & + & + & - & + & + & + & + & + & + & + & - & + & + & + & + \\
\hline $\mathrm{ZW}_{7}$ & + & - & - & - & - & - & - & - & - & - & - & - & - & - & - & - \\
\hline $\mathrm{ZW}_{8}$ & - & - & - & - & - & - & - & - & - & - & - & - & + & - & - & - \\
\hline $\mathrm{WH}_{1}{ }^{1}$ & - & - & - & - & + & - & - & - & + & - & - & - & + & + & + & + \\
\hline $\mathrm{WH}_{1}{ }^{2}$ & + & - & - & - & + & - & - & - & - & - & - & - & + & + & + & + \\
\hline $\mathrm{WH}_{1}{ }^{3}$ & + & + & - & - & + & + & + & + & - & - & - & - & + & + & + & + \\
\hline $\mathrm{WH}_{4}{ }^{1}$ & + & + & + & - & + & + & + & + & + & + & - & - & + & + & + & + \\
\hline $\mathrm{WH}_{4}{ }^{2}$ & - & - & - & - & + & + & + & + & - & - & - & - & + & - & - & - \\
\hline $\mathrm{WH}_{6}$ & + & + & - & - & + & + & + & + & + & - & - & - & + & + & + & - \\
\hline $\mathrm{AZ}_{1}$ & + & + & - & - & + & - & - & - & - & - & - & - & + & - & - & - \\
\hline $\mathrm{AZ}_{2}$ & + & - & - & - & + & + & + & + & + & - & - & - & + & + & + & + \\
\hline CAN & + & - & - & - & + & + & + & + & - & - & - & - & + & + & + & + \\
\hline WHn & - & - & - & - & + & - & - & - & - & - & - & - & + & - & - & - \\
\hline WHj & + & - & - & - & + & + & - & - & - & - & - & - & + & + & + & + \\
\hline $\mathrm{ZWn}$ & - & - & - & - & - & - & - & - & - & - & - & - & + & - & - & - \\
\hline WHm & - & - & - & - & + & - & - & - & - & - & - & - & - & - & - & - \\
\hline
\end{tabular}


乌拉特后旗。而乌拉特后旗的调查结果也说明了这 一点, 只有在水分条件较好的、由地表径流冲刷形成 的冲积沟底和两旁滩地上的沙冬青根系中发现根 瘤, 而其它沙冬青的根系中没有或极少着生有根瘤。 因此, 在降雨量少、蒸发强烈、气候干燥、热量资源丰 富的西北荒漠地带, 高温干旱成为沙冬青群落和微 生物生长发育的限制因子。

从不同分布区获得的沙冬青根瘤菌, 不管在耐 盐性、耐酸碱性、耐高温性,还是在抗生素抗性方面, 都表现出了很大的差异性。磴口县地处黄河两岸, 河水冲积形成的滩地和黄河故道有大量盐碱地, 从 此生态环境中分离得到的沙冬青根瘤菌具有极强的 抗盐性和抗酸碱性。沙冬青分布区夏季地面温度可 达 $60{ }^{\circ} \mathrm{C}$ 以上, 这赋予了沙冬青根瘤菌极强的抗热 性, 使其在 $60{ }^{\circ} \mathrm{C}$ 下处理 $10 \mathrm{~min}$ 后仍能生长。环境影 响表型, 进而导致种群的多样。进一步说明根瘤菌 与豆科植物的共生关系因区域地理环境的差异而具 多样性(陈文新等, 2004)。沙冬青根瘤菌抗逆性的 多样性可能是对相对恶劣的自然环境的一种适应, 是物种生态系统功能正常发挥的有效保障。

从结果可以看出, 虽然沙冬青根瘤菌在诸多抗 逆性方面存在着差异, 但是与已有的报道 (关桂兰 等, 1990, 1991)相比, 沙冬青根瘤菌无论在耐盐性、 耐酸碱性、耐高温性, 还是在抗生素抗性方面, 都表 现出了极强的抗性。在同一地区内, 沙冬青其伴生 植物的根瘤菌在抗盐碱性、抗高温等方面都具有很 强的抗性, 与沙冬青根瘤菌表现出了一定的相似性, 这说明生态环境对豆科植物-根瘤菌共生体有重要 的影响。

有关根瘤菌抗逆机制已有较多报道。在低渗环 境中 Rhizobium、Agrobacterium 和 Bradyrhizobium 的一 些菌株在壁膜间隙积累高浓度的葡聚糖, 为细菌提 供了调节细胞质体积、渗透压和壁膜间隙离子强度 的机制，而且已有相关基因的报道 (Miller \& Wood, 1996）。有些根瘤菌在高渗环境中积累甘氨酸、甜菜 碱来平衡细胞内外的渗透压。此外, 根瘤菌胞外多 糖的合成与渗透调节也密切相关。杨江科等 (2001) 研究了 $\mathrm{pH}$ 对根瘤菌生长状况和结瘤有影响。沙冬 青分布区缺水少氮的生境影响宿主与根瘤菌的共生 性质。不论是宿主还是细菌, 均经自然选择不断适 应干旱环境, 表现出耐盐、耐酸碱、耐高温的特性。 沙冬青分布区具有较高的光温生产潜力, 昼夜温差 较大, 旱生植物体内积累的碳水化合物较多, 从而为 固氮共生体提供了足够的能量。沙冬青根瘤菌较强
抗逆性的分子机理有待进一步研究。

沙冬青与根瘤菌的共生关系对沙冬青的生长发 育、优良性状的形成及其生物群落的发育和多样性 的维系具有重要作用。根瘤菌与宿主共生, 通过根 瘤共生体的固氮作用为宿主提供氮素。近年来的一 些研究还表明, 根瘤菌有利于宿主的抗病性和抗逆 性的提高: 定殖于植物根部的非病原体土生根瘤菌 产生诱导系统性抗性( ISR), ISR 需要茉莉酸 (JA) 和 乙烯介导的信号途径, 用以在远端的叶片组织中诱 导保护性的防御反应, 这种形式的防御不涉及致病 相关蛋白的积累, 也不需要水杨酸 (SA) (Buchanan et $a l ., 2000)$ 。植物抗氧化酶的亚细胞定位表明, 固氮 植物根瘤中含有抗坏血酸过氧化物酶 (APX)、脱氢 抗坏血酸还原酶( DHAR)、谷胱甘肽还原酶( GR)、单 脱氢抗坏血酸还原酶( MDHAR) 和超氧化物歧化酶 $(\mathrm{Cu} / \mathrm{ZnSOD})$ 等抗氧化酶(Buchanan et al.,2000)。沙 冬青分布区的高温、干早、低温和强光照等环境因子 都易导致氧化胁迫，而沙冬青根瘤中是否同样含有 以上抗氧化酶保护沙冬青根系及茎叶部分免受氧化 胁迫的破坏, 提高沙冬青抗逆性, 还需进一步的实验 证明。沙冬青高抗性与沙冬青根瘤菌的高抗性间可 能有密切联系, 其相互间的作用机理有待于进一步 的研究。

\section{参 考 文 献}

Allen ON, Allen EK (1981). The Legu minosae, a Source Book of Characteristics, Uses and Nodulation. The University of Wisconsin Press, Wisconsin, $707-727$.

Buchanan BB, Wilhelm G, Russell LJ (2000). Biochemitry \& Molecular Biology of Plants. American Socioty of Plant Physiogists, Rockvile, Maryland.

Chen WX, Li GS, Qi YL, Wang ET, Yuan HL, Li JL (1991). Rhizobium huakuii sp. nov. isolated from the root nodules of Astragalus sinisus. International Journal of Systematic Bacteriology, $41,275-280$.

Chen WX(陈文新), Wang ET (汪恩涛), Chen WF (陈文峰) (2004). The relationship between the symbiotic promiscuity of rhizobia and legumes and their geographical environments. Scientia Agriculture Sinica (中国农业科学) ,37,81-86. (in Chinese with English abstract)

Chen X(陈曦), Lu CF (卢存福), Jiang XN(蒋湘宁), Wang SS (王沙生) (2002). New progress in the study of plant antifreeze proteins and their genetic engineering. Journal of Beijing Forestry University (北京林业大学学报), 24,94-98. (in Chinese with English abstract)

Delectis Florae Reipublicae Sinecae Agendae Academiae Sinicae Edita(中国植物志编辑委员会) (1998). Flora Reipublicae 
Popularis Sinica (中国植物志), Science Press, Beijing, 395. Guan GL(关桂兰), Su Y (苏云), Wang WW (王卫卫) (1990).

Hydrogen uptake and nitrogen fixing of noduled leguminous plants in Xinjiang arid area. Arid Zone Research(干旱区研究) ,7,9 14. (in Chinese with English abstract)

Guan GL (关桂兰), Wang WW (王卫卫), Yang YS (杨玉锁) (1991). Nitrogen Fixation Biological Resource in Xinjiang Arid Region (新疆干旱区生物固氮资源). Science Press, Beijing. (in Chinese)

Han SF(韩素芬), Chen JR(陈景荣), Xie WJ(谢文娟) (1996). Experiment on the inoculation test between rhizobia from woody legumes and four leguminous species. Forest Research (林业科 学研究) ,6,610-615. (in Chinese with English abstract)

Liu GH(刘果厚) (1998). Study on the endangered reasons of Ammopiptanthus mongolicus in the desert of alashan. Bulletin of Botanical Research (植物研究) , 18,341 - 345. (in Chinese with English abstract)

Liu JQ(刘家琼), Qiu MX(邱明新), Yang K(杨口), Shi QH(石 庆辉) (1995). Studies on the plant community of Ammopiptanthus mongolicus. Journal of Desert Research (中国沙漠) , 15,109 - 115. (in Chinese with English abstract)

Miller KJ, Wood JM(1996) . Osmoadaptation by rhizosphere bacteria. Annual Review of Microbiology, 50,101-136.

Subba Rao NS (1999). Soil Microbiology 4th edn. Soil Microorganisms and Plant Growth. USA. Science Publishers Inc., Washington.
Vincent JM (1970) . A Manual for the Practical Study of Root Nodule Bacteria. IBP Handbook No.15. Blackwell Scientific Publications, Oxford.

Wang J(王静), Ma YZ(马玉珍), Shi QL(史清亮), Chen WX(陈 文新), Cai XM(蔡雪梅) (1999). Study on diversity and particularity of rhizobia resources in Shanxi. Chinese Journal of Applied \& Environmental Biology (应用与环境生物学报), 5,79-84. (in Chinese with English abstract)

Wang QS(王庆锁), Li Y (李勇), Zhang LZ(张灵芝) (1995).A summary of studies on the rare and endangered Ammopiptanthus mongolicus. Chinese Biodiversity (生物多样性) , 3, 153 - 156 . (in Chinese with English abstract)

Wang WW(王卫卫), Guan GL(关桂兰), Guo PX (郭沛新), Dong WC(董文彩), Li Y (李渘) (1995). Investigations on the symbiotic nitrogen-fixing resource of legume in Tianshui, Gansu. Arid Zone Research (干旱区研究), 12,7-10. (in Chinese with English abstract)

Yang JK(杨江科), Zhou Q(周琴), Zhou JC(周俊初) (2001). Effect of $\mathrm{pH}$ on nodulation of soybean rhizobia from Weifang and Huayuankou soils. Chinese Journal of Applied Ecology (应用生 态学报),12,639-640. (in Chinese with English abstract)

Zhou YJ(周宜君), Liu CL(刘春兰), Feng JZ(冯金朝), Jia XH (贾晓红)（2001）. Advances of drought-resistance and frigid-resistance mechanism research on Ammopiptanthus mongolicus. Journal of Desert Research (中国沙漠), 21,312 - 316. (in Chinese with English abstract) 